Ambiente \& Água - An Interdisciplinary Journal of Applied Science
ISSN 1980-993X - doi:10.4136/1980-993X
www.ambi-agua.net
E-mail: ambi-agua@agro.unitau.br

\title{
Parámetros del modelo de infiltración de Horton obtenidos mediante el uso de un simulador de lluvia, Córdoba, Argentina
}

\author{
doi: 10.4136/ambi-agua.1320 \\ Received: 18 Feb. 2014; Accepted: 08 Mar 2014 \\ Juan Francisco Weber \\ Universidad Tecnológica Nacional, Córdoba, Argentina \\ Departamento de Ingeniería Civil \\ Autor correspondiente: e-mail: jweber@civil.frc.utn.edu.ar
}

\section{RESUMEN}

Se presentan los resultados obtenidos del ajuste de los parámetros del modelo de infiltración de Horton, a partir de los datos experimentales recopilados en una campaña de medición in situ de la capacidad de infiltración en distintos sitios de la ciudad de Córdoba (Argentina). Como instrumento se utilizó un microsimulador de lluvia portátil diseñado y construido en el Laboratorio de Hidráulica, UTN-Facultad Córdoba. Este equipo, que es completamente desarmable y define una parcela de ensayo de $1 \mathrm{~m}^{2}$, permite generar lluvias de intensidades comprendidas entre los 65 y $120 \mathrm{~mm} / \mathrm{h}$. La selección de los sitios de ensayo respondió a dos condiciones: el tipo y el uso del suelo. A su vez, se consideraron distintas condiciones de humedad inicial. Los valores medidos fueron digitalizados y procesados, construyéndose las curvas de infiltración acumulada y tasa de infiltración en función del tiempo. A partir de estos valores, se ajustaron los parámetros del modelo de Horton. Los parámetros así obtenidos, que superan en calidad a los determinados en campañas anteriores utilizando la técnica del infiltrómetro de doble anillo, serán de utilidad en tareas de diseño hidrológico urbano, y representan un aporte original al conocimiento de la respuesta hidrológica de los suelos urbanos de Córdoba. La propuesta podrá repetirse en otros sitios ampliando de esa forma su significación científica.

Palabras-clave: hidrología experimental, mediciones hidrológicas, hidrología urbana.

\section{Parâmetros do modelo de infiltração de Horton desenvolvidos utilizando-se um simulador de chuva, Córdoba, Argentina}

\section{RESUMO}

Neste artigo são apresentados resultados do ajuste dos parâmetros do modelo de infiltração de Horton para vários locais na cidade de Córdoba (Argentina), com dados experimentais obtidos em uma campanha de medição de campo da capacidade de infiltração, em que foi utilizado um simulador de chuva portátil, projetado e construído no Laboratório de Hidráulica, UTN - Facultad Córdoba. Esse equipamento, que é completamente desmontável e define um plano de teste de $1 \mathrm{~m}^{2}$, pode gerar intensidades de precipitação que variam entre 65 e $120 \mathrm{~mm} / \mathrm{h}$. A seleção dos locais para obtenção das amostras experimentais foi relacionada a duas condições: o tipo e o uso do solo, tendo sido considerada a condição inicial de umidade do local. Os valores medidos foram digitalizados e processados, e traçadas as curvas de infiltração cumulativa e taxa de infiltração. A partir desses valores obtidos experimentalmente foram ajustados os parâmetros do modelo de Horton. E os 
resultados finais mostraram que os parâmetros obtidos mostraram-se melhor ajustados do que aqueles determinados com o uso da técnica do infiltrômetro duplo, apresentando assim, melhor utilidade para trabalhos de hidrologia urbana para solos urbanos da cidade de Córdoba, e a sua repetição para outros locais poderá ampliar o significado científico da proposta.

Palavras-chave: hidrologia experimental, medições hidrológicas, hidrologia urbana.

\title{
Parameters of Horton's infiltration model obtained through the use of a rainfall simulator, Córdoba, Argentina
}

\begin{abstract}
This study presents the results of a parameter fit of the Horton infiltration model at various sites in the city of Córdoba, Argentina. The work is based upon experimental data collected in field measurements of infiltration capacity. The study employed a portable rainfall simulator that was designed and built at the Hydraulics Laboratory, UTN - Facultad Córdoba. This equipment, which is completely detachable and defines a test plot of $1 \mathrm{~m}^{2}$, can generate rainfall intensities which vary between 65 and $120 \mathrm{~mm} / \mathrm{h}$. Test site selection depended upon soil type and land use. Additionally, different initial moisture conditions were considered. The measured values were digitized and processed, and cumulative infiltration and infiltration rate curves were plotted. From these values, the parameters of Horton's model were adjusted. The quality of these parameters exceeded those of previous studies that used the double ring infiltrometer technique, and will be useful in urban hydrology design works. They represent an original contribution to knowledge of the hydrological response of urban soils in Córdoba. The proposal may be repeated elsewhere in order to expand its scientific significance.
\end{abstract}

Keywords: experimental hydrology, hydrological measurements, urban hydrology.

\section{INTRODUCCIÓN}

En trabajos ingenieriles vinculados al diseño y cálculo de obras de desagüe pluvial urbano, el proyectista se ve en la obligación, a la hora de cuantificar los caudales de aporte, a considerar dos tipos de superficies en las cuencas: las impermeables y las permeables. Las primeras, sin duda provocan un alto impacto en el volumen de crecidas y una disminución del tiempo de respuesta de la cuenca (Tucci, 2001). En cuanto a las segundas, se han impuesto en la práctica un conjunto de modelos de uso generalizado a la hora de cuantificar la fracción de la precipitación de escurrimiento directo, y por tanto, la fracción infiltrada; estos modelos, de carácter conceptual (Ravi y Williams, 1998), intentan simplificar el abordaje riguroso del problema, representado por la ecuación de Richards (Chow et al., 1994), y por tanto son los utilizados más ampliamente en la práctica hidrológica.

Una de las principales dificultades que encuentra el modelador a la hora de aplicar estos modelos de infiltración es la de estimar los parámetros de los mismos, tratando de lograr una adecuada representación de la realidad. Lamentablemente, existen grandes dificultades a la hora de disponer de datos medidos de infiltración en áreas permeables urbanas en la República Argentina. En la ciudad de Córdoba, por ejemplo, recién se encara una tarea sistemática de producción de información experimental al respecto a partir del trabajo de Weber et al. (2005), a través una campaña de obtención de datos experimentales de infiltración en suelos de la ciudad de Córdoba, mediante la técnica del infiltrómetro de doble anillo. Este procedimiento de ensayo permite obtener mediciones directas de infiltración en áreas reducidas (puntuales). Son conocidas las severas limitaciones que presentan las mediciones así determinadas, fundamentalmente en relación a la falta de representatividad del proceso lluvioso en la técnica de ensayo, por un lado, y a la fuerte alteración de la superficie 
del terreno debido al hincado de los anillos; estas limitaciones conllevan a errores experimentales difíciles de cuantificar.

Con el objetivo de obtener una caracterización experimental más verosímil del proceso hidrológico de la infiltración, se propuso utilizar como instrumento de ensayo un simulador de lluvia portátil de desarrollo propio; se esperarían como beneficios inmediatos: una mejor simulación del proceso lluvia - infiltración (debido a su mayor similitud con el proceso real), y una menor alteración de la parcela de ensayo; es decir, se intenta subsanar las limitaciones del procedimiento del infiltrómetro de doble anillo anteriormente utilizado.

\section{MATERIALES Y MÉTODOS}

\subsection{El simulador de lluvia}

Los simuladores de lluvia son instrumentos de investigación diseñados para aplicar agua de forma similar a los episodios tormentosos naturales. Son útiles para obtener datos de erosión, infiltración, escorrentía superficial y transporte de sedimentos. No obstante, las características de la lluvia natural deben ser simuladas de forma adecuada, los datos obtenidos deben ser analizados cuidadosamente y se debe efectuar una buena interpretación de los resultados para obtener información fiable en las condiciones para las que ha sido aplicable la lluvia (Benito et al., 2003).

Uno de los antecedentes de mayor peso en el diseño del simulador de lluvia utilizado fue el precedente local de un instrumento diseñado por Irurtia y Mon (1994); a partir de un modelo de Kamphorst (1987). Este microsimulador de lluvia fue luego modificado por Aoki y Sereno (1999), y se encuentra actualmente en uso en la Facultad de Agronomía de la Universidad Nacional de Córdoba.

El formador utilizado se compone de una aguja hipodérmica introducida en tubo rígido, el cual se obtiene de cortar su propio estuche, al ras de dicha aguja. Los formadores ensayados correspondieron a un mismo diámetro de la aguja, $0.8 \mathrm{~mm}$, y tres longitudes diferentes, $25.4 \mathrm{~mm}, 38.1 \mathrm{~mm}, 50.8 \mathrm{~mm}$, las que corresponden a $1,1 \frac{1}{2}$ y 2 pulgadas respectivamente.

Se realizó una serie de mediciones de laboratorio para determinar el comportamiento hidráulico de estos formadores. En base a esta información se decidió colocar un total de 289 formadores, distribuidos en 17 filas por 17 columnas, y separados a una distancia de 5,30 cm entre ejes, cubriendo de esta manera cada formador, un área aproximada de $28,10 \mathrm{~cm}^{2}$ sobre la base de una batea de $96 \mathrm{~cm}$ x $96 \mathrm{~cm}$ de lado. El tipo de formador seleccionado fue el $\mathrm{N}^{\mathrm{o}} 3$, correspondiente a la aguja de 2 pulgadas, ya que fue el que presentó las menores intensidades.

El equipo (Figura 1) consta básicamente de una estructura metálica, de caño de acero de sección cuadrada, formando un prisma cuadrado de $2 \mathrm{~m}$ de altura y $1 \mathrm{~m}$ de lado. Este prisma se forma a través de cuatro columnas y cerrado por medio de cortinas rompevientos. Las columnas sostienen el sistema de alimentación de agua que está compuesto por dos tanques; uno de alimentación, solamente y el otro de alimentación y regulación de intensidad.

La regulación de la intensidad se produce a través de un flotante (Achutegui et al., 1996). Debajo del sistema de alimentación de agua se encuentra la batea con los 289 formadores de gotas, que producen la lluvia simulada. La base de este prisma está constituida por un marco cuadrado de hierro, de $1 \mathrm{~m}$ de lado y $0.10 \mathrm{~m}$ de altura, con vertederos en sus cuatro lados y canales para el transporte del agua escurrida. Este marco de hierro, en su posición de trabajo es hincado en el suelo, constituyendo así las paredes límites de la parcela de medición. El peso total del equipo armado es de $110.60 \mathrm{~kg}$; el peso del fluido puede llegar hasta los $100 \mathrm{~kg}$. 


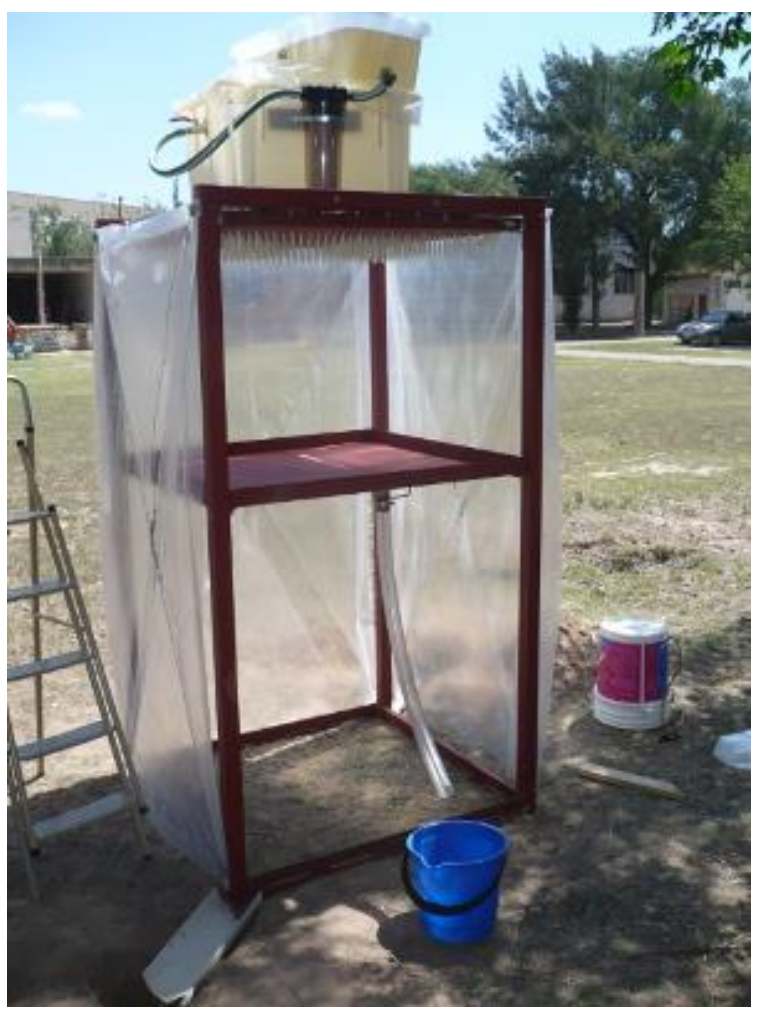

Figura 1. Simulador de lluvia en campaña.

\subsection{Tareas de calibración}

Para la calibración se utilizaron cinco probetas graduadas de $1000 \mathrm{ml}$, con divisiones de $10 \mathrm{ml}$ cada una. En su parte superior se les colocó un embudo de $32 \mathrm{~cm}$ de diámetro exterior y $31 \mathrm{~cm}$ de diámetro interior, funcionando el conjunto como un pluviómetro. Se midió el tiempo que tardó en llenarse un volumen fijo de $850 \mathrm{ml}$ en cada pluviómetro para las diferentes cargas estudiadas. Estas fueron $11 \mathrm{~cm}, 13 \mathrm{~cm}, 15 \mathrm{~cm}, 17,5 \mathrm{~cm}, 20 \mathrm{~cm}, 22,5 \mathrm{~cm}$ y $25 \mathrm{~cm}$.

Cada uno de los puntos fue medido simultáneamente durante un funcionamiento continuo del simulador de lluvia de aproximadamente $2 \mathrm{~h}$ por ensayo, obteniéndose de esta manera 10 datos por pluviómetro, y una muestra total de 50 datos para cada carga analizada. Esto representa un total de 350 datos obtenidos. Los resultados de estas mediciones debieron ser corregidos por el error sistemático introducido en el posicionamiento de los pluviómetros, que produjo que el número de formadores capturados variara entre 23 y 25.

Se verificó la normalidad de la distribución de las observaciones, estandarizando cada serie. Con los valores medios de intensidad obtenidos para cada carga, se trazó la curva de ajuste indicada en la Figura 2. En esta figura, las curvas que rodean a la regresión potencial corresponden a bandas de $99 \%$ y $90 \%$ de certidumbre, respectivamente, con un coeficiente de determinación $\mathrm{R}^{2}=0.997$ de acuerdo con la Ecuación 1 .

$$
i=15,15 h^{0.63}
$$

en que:

$i$ - es la intensidad de lluvia producida (en $\mathrm{mm} / \mathrm{h}$ ) y

$h$ - es la carga (en cm) sobre los formadores.

Del análisis estadístico de los datos, se pudo concluir que los valores de intensidad obtenidos a partir de la Ecuación 1 tienen una incertidumbre asociada de $3 \mathrm{~mm} / \mathrm{h}$, lo cual representa un error de entre el 3 y el $5 \%$ de la intensidad producida. 


\subsection{Trabajos experimentales}

Para la definición de los sitios de ensayo se consideró que las dos variables a priori más influyentes sobre los resultados a obtener eran el tipo de suelo y el uso del suelo (Weber et al., 2005). Con respecto al tipo, más que a una clasificación geotécnica se asumió que una clasificación de características regionales resultaría con mayor representatividad hidrológica. Para ello, se fijó como criterio la clasificación de ambientes geomorfológicos desarrollada por Quintana Salvat y Barbeito (1994). Este trabajo detectó básicamente dos ambientes diferenciados en el ejido de la ciudad de Córdoba: la planicie loessoide (correspondiente a un suelo del tipo limo arenoso, de gran cohesión en condiciones de baja humedad) y la terraza de inundación del antiguo cauce del río Suquía (Primero), la cual fue a su vez clasificada por los autores citados en tres subambientes: terraza baja, media y alta, según su cercanía al actual cauce del río que atraviesa la ciudad.

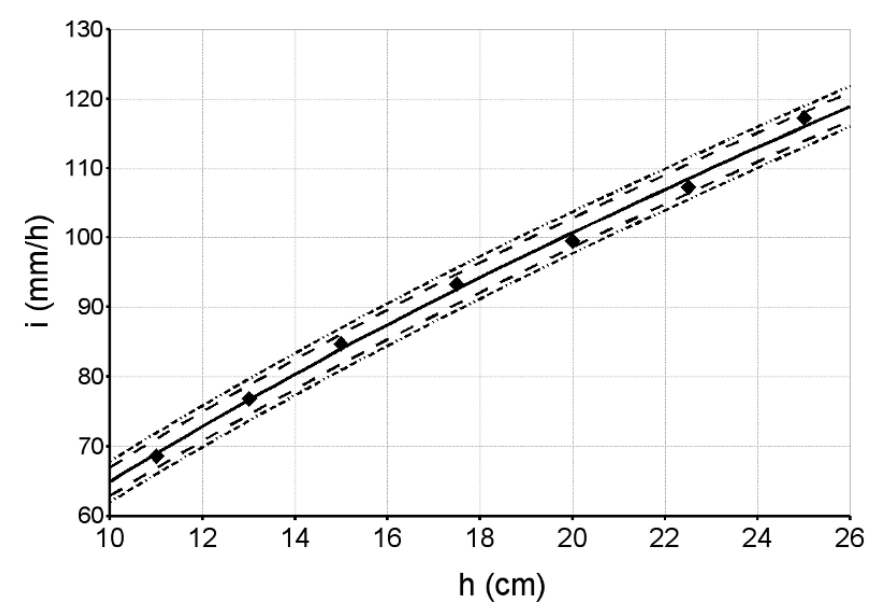

Figura 2. Curva de calibración del simulador de lluvia.

En cuanto al uso del suelo, se distinguió básicamente en tres tipos fundamentales: los patios y jardines de viviendas residenciales (espacios verdes con bajo tránsito), las plazas, parques y paseos públicos (espacios verdes con medio tránsito), y finalmente las calles no pavimentadas (calles de tierra). El principal criterio de selección se basó en la intensidad del tránsito, tanto peatonal como vehicular, debido a su impacto directo en la capacidad del infiltración del suelo. Además, se agregó como punto de interés especial el lecho de una laguna de detención en la zona norte de la ciudad.

El Cuadro 1 muestra los sitios de ensayo seleccionados clasificados según el uso del suelo y el ambiente geomorfológico. Como puede verse, no fue posible conseguir terreno disponible para realizar el ensayo correspondiente a espacios verdes de bajo tránsito en la terraza media del río Suquía, debido a que este ambiente cubre un área relativamente pequeña y fuertemente urbanizada de la ciudad. En total se ensayaron 12 sitios a lo largo del ejido urbano de la ciudad.

En cada uno de los sitios seleccionados se realizaron tres ensayos con el simulador de lluvia, bajo intensidades relativamente constantes (del orden de los $70 \mathrm{~mm} / \mathrm{h}$ ) con diferentes condiciones iniciales de humedad, las cuales fueron obtenidas o bien por las condiciones climáticas imperantes o bien los terrenos fueron humedecidos (regados) previamente a partir de condiciones secas. Además, en la mayoría de los sitios se realizó simultáneamente el ensayo clásico de infiltración mediante el infiltrómetro de doble anillo, como control y contraste de las mediciones efectuadas. La campaña de medición se extendió durante siete meses e implicó el trabajo de tres personas en forma simultánea, realizando entre dos y tres ensayos por semana. 
Cuadro 1. Sitios de ensayo.

\begin{tabular}{|c|c|c|c|}
\hline \multirow{2}{*}{$\begin{array}{l}\text { Tipo de suelo } \\
\text { (ambiente } \\
\text { geomorfológico) }\end{array}$} & \multicolumn{3}{|c|}{ Uso del suelo } \\
\hline & $\begin{array}{l}\text { Espacios verdes } \\
\text { (bajo tránsito) }\end{array}$ & $\begin{array}{l}\text { Espacios verdes } \\
\text { (medio tránsito) }\end{array}$ & $\begin{array}{c}\text { Calles no pavimentadas (alto } \\
\text { tránsito) }\end{array}$ \\
\hline Terraza alta & ITS Villada & ITS Villada & ITS Villada \\
\hline Terraza media & & Parque General Paz & $\begin{array}{l}\text { Justo Liebig } 5940-\mathrm{B}^{\circ} \text { Villa } \\
\text { Belgrano }\end{array}$ \\
\hline Terraza baja & Torres de $\mathrm{B}^{\mathrm{o}}$ Junior & $\begin{array}{l}\text { Parque de las } \\
\text { Naciones }\end{array}$ & $\begin{array}{l}\text { Carlos Gauss } 4619-\mathrm{B}^{\circ} \\
\text { Villa Belgrano }\end{array}$ \\
\hline $\begin{array}{l}\text { Planicie } \\
\text { loessoide }\end{array}$ & $\begin{array}{l}\text { L. Suárez de } \\
\text { Figueroa }-\mathrm{B}^{\circ} \mathrm{M} \text {. de } \\
\text { Sobremonte }\end{array}$ & $\begin{array}{l}\text { parque UTN } \\
\text { Laguna de detención }\end{array}$ & $\begin{array}{l}\text { Hugo Miatello } 4600-\mathrm{B}^{\circ} \\
\text { Poeta Lugones }\end{array}$ \\
\hline
\end{tabular}

La duración de los ensayos fue variable, comprendida en general entre 1.5 y 2 h, dependiendo del comportamiento observado in situ del proceso de infiltración, en cuanto a la velocidad con que se alcanzó la condición de régimen. El número y frecuencia de las observaciones también fue dependiente de las condiciones locales encontradas, observándose una menor frecuencia de medición en el simulador (y por tanto, una mayor frecuencia en el infiltrómetro) en los suelos de mayor capacidad de infiltración. Para facilitar la tarea de medición, se seleccionó en cada caso el volumen de registro, el cual varió desde 50 hasta $500 \mathrm{~cm}^{3}$.

Por otro lado, en todos los casos se midió la tasa de captura de las cortinas laterales, la cual fue considerada en el balance hidrológico a la hora del procesamiento de la información.

Antes del inicio de cada ensayo, se extrajeron muestras de suelo para la determinación del contenido de humedad antecedente. El contenido de humedad fue determinado gravimétricamente, a través de la extracción de muestras de suelo a $10 \mathrm{~cm}$ de profundidad, secándolas en estufa a $110{ }^{\circ} \mathrm{C}$ y pesando la muestra seca. Para relacionar esta humedad gravimétrica con la humedad volumétrica $\theta$ se utilizó la densidad (peso unitario) del suelo. Este valor fue estimado a partir de las observaciones in situ realizadas en tareas anteriores en cada uno de los ambientes geomorfológicos descriptos (Weber et al., 2005).

Los datos así obtenidos fueron procesados utilizando la planilla de cálculo Microsoft Excel, que permitió no sólo la representación gráfica de la información recopilada, sino también las tareas de ajuste de parámetros que se describirán posteriormente. 


\subsection{El modelo de Horton}

Horton (1939) propuso, en base a numerosas observaciones experimentales, las Ecuaciones 2 y 3 para estimar la tasa de infiltración y la infiltración acumulada.

$$
\begin{aligned}
& f(t)=f_{b}+\left(f_{0}-f_{b}\right) e^{-k t} \\
& F(t)=f_{b} t+\frac{f_{0}-f_{b}}{k}\left(1-e^{-k t}\right)
\end{aligned}
$$

en que corresponde a un modelo de tres parámetros:

$$
\begin{aligned}
& f_{b}, f_{0} \text { y } k \text {, donde } \\
& f_{b} \text { - es la tasa base de infiltración (capacidad mínima de infiltración), } \\
& f_{0} \text { - es la tasa inicial de infiltración y } \\
& k \text { - un parámetro de forma. }
\end{aligned}
$$

En base a la información recopilada en campaña, se realizó el ajuste de los parámetros de este modelo, buscando la minimización de la función objetivo F.O. representada por la suma de las desviaciones cuadráticas entre los n valores de infiltración acumulada observados $\left(F_{i}\right)$ y calculados $\left(F_{i}^{c}\right)$, para cada sitio de ensayo y cada condición inicial de humedad, de acuerdo con la Ecuación 4.

$$
\text { F.O. }=\sum_{i=1}^{n}\left(F_{i}-F_{i}^{c}\right)^{2}
$$

El proceso se automatizó mediante un código especialmente desarrollado en GNU Octave (Eaton et al., 2007) que permitió ajustar para cada uno de los 34 archivos de datos, los parámetros óptimos del modelo de Horton a través del algoritmo de optimización multidimensional no restringida de Nelder-Mead (Mathews y Fink, 2004).

\section{RESULTADOS Y DISCUSIÓN}

De este modo se obtuvieron 34 juegos de parámetros, junto con el valor del coeficiente de determinación $\mathrm{R}^{2}$, el cual es una medida del grado de ajuste alcanzado. En la Tabla 1 se presentan los parámetros así ajustados. En esa tabla puede apreciarse que a pesar de haberse implementado una optimización no restringida, en todos los casos los parámetros obtenidos cumplen la condición $f_{0}>f_{b}$. Pueden observarse, además, los valores relativamente altos del coeficiente de determinación $\mathrm{R}^{2}$. Este estadístico tomó valores comprendidos entre $0.9950 \mathrm{y}$ 0.9999, con una media de 0.9990. Su significancia estadística es muy alta, pudiendo no rechazarse la hipótesis nula aún para valores de $\alpha$ tan bajos como $1 \cdot 10^{-115}$ (esto debido principalmente al elevado número de grados de libertad, 173 en promedio).

En la Tabla 2 se indican los valores promedio (y el rango de variación) para los parámetros del modelo de Horton, clasificados según el uso del suelo. Puede observarse un rango bastante amplio de valores para los tres parámetros.

En la Figura 3 se presenta, a título de ejemplo, la infiltración acumulada medida en un ensayo en particular ( $2^{\circ}$ medición - Marqués de Sobremonte) junto con los valores predichos por el modelo de Horton. 
En la Figura 4 puede verse la correspondencia entre los valores observados y predichos por el modelo de Horton de infiltración acumulada; la recta de regresión de acuerdo con la Ecuación 5 entre estos conjuntos de datos (5898 puntos) es:

$$
F_{c}=1.001245 F_{m}-0.035053
$$

en que:

$F_{c}$ - es la infiltración acumulada calculada por Horton y

$F_{m}$ - la observada.

Puede verse la proximidad de esta recta a la identidad $(\mathrm{Fc}=\mathrm{Fm})$, correspondiente al ajuste perfecto. El valor medio de los errores $F_{c}-F_{m}$ resultó en $-0.0056 \mathrm{~mm}$; mientras que el valor medio del valor absoluto de estos errores es de $0.25 \mathrm{~mm}$, con un máximo de $1.85 \mathrm{~mm}$.

Los mayores errores porcentuales en la estimación de la infiltración ocurren (con un máximo del 52.6\%) para valores bajos de infiltración, correspondientes a los primeros instantes del ensayo, tendiendo a disminuir en valor absoluto a medida que transcurre el mismo, tal como se observa en la Figura 5, donde se representa el conjunto completo de observaciones realizadas. El error (en valor absoluto) porcentual promedio en la estimación por Horton resultó en $1.9 \%$.

Se analizó la posible correlación entre los parámetros ajustados del modelo. Se observó que los parámetros $f_{0}$ y $k$ presentaron cierta correlación $\left(\mathrm{R}^{2}=0.794\right)$ tal como había sido observado por Weber et al. (2005) a partir de mediciones realizadas con infiltrómetros de doble anillo. En la Figura 6 se observa un gráfico de dispersión entre estas dos variables, junto con la recta de regresión de acuerdo con la Ecuación 6, cuya expresión es:

$$
f_{0}(\mathrm{~mm} / \mathrm{h})=37.6+3.36 k(1 / h)
$$

Es posible sustituir el valor de $f_{0}$ en la Ecuación 3 por el dado en la Ecuación 6, transformando de este modo al modelo en biparamétrico. Esta sustitución da lugar a un incremento en los errores de estimación. El valor medio de estos errores resultó en $0.2 \mathrm{~mm}$; mientras que el valor medio del valor absoluto de estos errores es de $1.9 \mathrm{~mm}$, con un máximo de $8.6 \mathrm{~mm}$. Los valores porcentuales correspondientes a los dos últimos resultaron, respectivamente, $11.9 \%$ y 129\%. En ausencia de otra información, la Ecuación 6 puede ser utilizada, en el área de estudio, para obtener una estimación de $f_{0}$ a partir de $k$ (o viceversa).

Por otra parte es de tener presente que la intensidad de la propia lluvia generada tiene un error de $\pm 3 \mathrm{~mm} / \mathrm{h}$ (Weber et al., 2010). Esto implica que para una duración típica del ensayo de $1.5 \mathrm{~h}$, este valor se transforma en una incertidumbre de $\pm 4.5 \mathrm{~mm}$ en la medición de la infiltración acumulada, muy superior en general a los errores asociados al uso de la Ecuación 6. Esto puede observarse a título de ejemplo en la Figura 7, donde se representa la infiltración acumulada medida (línea negra continua) junto con sus bandas de incertidumbre (líneas de trazo), superpuestas a las infiltraciones predichas por el modelo de Horton con sus tres parámetros (puntos rojos) o con la sustitución de la Ecuación 6 (puntos azules), para un ensayo en particular ( $2^{\circ}$ medición - casa $\mathrm{B}^{\mathbf{o}}$ Marqués de Sobremonte). 
Tabla 1. Parámetros ajustados del modelo de Horton $\left(f_{o}\right.$ y $f_{b}$ en $\mathrm{mm} / \mathrm{h}, k$ en $\left.1 / \mathrm{h}\right)$.

\begin{tabular}{|c|c|c|c|c|c|c|c|}
\hline Uso & Sitio & & ición & $2^{\circ} n$ & lición & $3^{\circ} \mathrm{m}$ & ición \\
\hline \multirow{16}{*}{$\begin{array}{c}\text { Calles } \\
\text { De } \\
\text { tierra }\end{array}$} & \multirow{4}{*}{ Gauss 4619} & $f 0=$ & 89.61 & $f 0=$ & 123.28 & $f 0=$ & 18.84 \\
\hline & & $\mathrm{fb}=$ & 14.91 & $\mathrm{fb}=$ & 21.58 & $\mathrm{fb}=$ & 15.07 \\
\hline & & $\mathrm{k}=$ & 9.42 & $\mathrm{k}=$ & 16.20 & $\mathrm{k}=$ & 3.89 \\
\hline & & $\mathrm{R}^{2}=$ & 0.9993 & $\mathrm{R}^{2}=$ & 0.9997 & $\mathrm{R}^{2}=$ & 0.9983 \\
\hline & \multirow{4}{*}{ Miatello 4600} & $\mathrm{f0}=$ & 83.45 & $\mathrm{f0}=$ & 69.29 & $\mathrm{fO}=$ & 28.17 \\
\hline & & $\mathrm{fb}=$ & 27.36 & $\mathrm{fb}=$ & 23.16 & $\mathrm{fb}=$ & 19.66 \\
\hline & & $\mathrm{k}=$ & 13.06 & $\mathrm{k}=$ & 13.87 & $\mathrm{k}=$ & 4.78 \\
\hline & & $\mathrm{R}^{2}=$ & 0.9993 & $\mathrm{R}^{2}=$ & 0.9998 & $\mathrm{R}^{2}=$ & 0.9990 \\
\hline & \multirow{4}{*}{ Liebig 5940} & $\mathrm{f} 0=$ & 60.12 & $\mathrm{f0}=$ & 31.22 & $\mathrm{f0}=$ & 54.74 \\
\hline & & $\mathrm{fb}=$ & 36.58 & $\mathrm{fb}=$ & 31.15 & $\mathrm{fb}=$ & 20.28 \\
\hline & & $\mathrm{k}=$ & 6.94 & $\mathrm{k}=$ & 6.89 & $\mathrm{k}=$ & 6.38 \\
\hline & & $\mathrm{R}^{2}=$ & 0.9998 & $\mathrm{R}^{2}=$ & 0.9991 & $\mathrm{R}^{2}=$ & 0.9995 \\
\hline & \multirow{4}{*}{ ITS Villada } & $\mathrm{f} 0=$ & 96.95 & & & $\mathrm{fO}=$ & 90.39 \\
\hline & & $\mathrm{fb}=$ & 12.46 & & & $\mathrm{fb}=$ & 27.18 \\
\hline & & $\mathrm{k}=$ & 11.26 & & & $\mathrm{k}=$ & 16.04 \\
\hline & & $\mathrm{R}^{2}=$ & 0.9981 & & & $\mathrm{R}^{2}=$ & 0.9994 \\
\hline \multirow{20}{*}{ parques } & \multirow{4}{*}{ Parque Gral. Paz } & $\mathrm{fO}=$ & 101.59 & $\mathrm{f0}=$ & 208.58 & $\mathrm{f0}=$ & 91.49 \\
\hline & & $\mathrm{fb}=$ & 47.78 & $\mathrm{fb}=$ & 25.73 & $\mathrm{fb}=$ & 43.54 \\
\hline & & $\mathrm{k}=$ & 17.44 & $\mathrm{k}=$ & 51.12 & $\mathrm{k}=$ & 17.01 \\
\hline & & $\mathrm{R}^{2}=$ & 0.9997 & $\mathrm{R}^{2}=$ & 0.9986 & $\mathrm{R}^{2}=$ & 0.9991 \\
\hline & \multirow{4}{*}{ parque ITS Villada } & $\mathrm{f} 0=$ & 64.63 & $\mathrm{fO}=$ & 36.37 & $\mathrm{f} 0=$ & 66.80 \\
\hline & & $\mathrm{fb}=$ & 26.69 & $\mathrm{fb}=$ & 30.01 & $\mathrm{fb}=$ & 26.48 \\
\hline & & $\mathrm{k}=$ & 8.88 & $\mathrm{k}=$ & 5.98 & $\mathrm{k}=$ & 7.84 \\
\hline & & $\mathrm{R}^{2}=$ & 0.9999 & $\mathrm{R}^{2}=$ & 0.9993 & $\mathrm{R}^{2}=$ & 0.9990 \\
\hline & \multirow{4}{*}{ Laguna de retencion } & $\mathrm{f} 0=$ & 36.61 & $\mathrm{f0}=$ & 85.39 & $\mathrm{f0}=$ & 104.14 \\
\hline & & $\mathrm{fb}=$ & 14.69 & $\mathrm{fb}=$ & 25.10 & $\mathrm{fb}=$ & 28.82 \\
\hline & & $\mathrm{k}=$ & 4.65 & $\mathrm{k}=$ & 14.60 & $\mathrm{k}=$ & 16.35 \\
\hline & & $\mathrm{R}^{2}=$ & 0.9970 & $\mathrm{R}^{2}=$ & 0.9995 & $\mathrm{R}^{2}=$ & 0.9999 \\
\hline & \multirow{4}{*}{ Parque de las Naciones } & $\mathrm{f} 0=$ & 72.32 & & & $\mathrm{f0}=$ & 148.60 \\
\hline & & $\mathrm{fb}=$ & 42.76 & & & $\mathrm{fb}=$ & 30.55 \\
\hline & & $\mathrm{k}=$ & 5.72 & & & $\mathrm{k}=$ & 35.28 \\
\hline & & $\mathrm{R}^{2}=$ & 0.9999 & & & $\mathrm{R}^{2}=$ & 0.9996 \\
\hline & \multirow{4}{*}{ UTN } & $\mathrm{f} 0=$ & 53.97 & $\mathrm{f} 0=$ & 62.96 & $\mathrm{f} 0=$ & 108.63 \\
\hline & & $\mathrm{fb}=$ & 26.27 & $\mathrm{fb}=$ & 22.69 & $\mathrm{fb}=$ & 34.20 \\
\hline & & $\mathrm{k}=$ & 4.04 & $\mathrm{k}=$ & 4.24 & $\mathrm{k}=$ & 24.50 \\
\hline & & $\mathrm{R}^{2}=$ & 0.9996 & $\mathrm{R}^{2}=$ & 0.9996 & $\mathrm{R}^{2}=$ & 0.9989 \\
\hline \multirow{12}{*}{ residencias } & \multirow{4}{*}{ casa Marques de Sobremonte } & $\mathrm{f} 0=$ & 74.56 & $\mathrm{f} 0=$ & 54.27 & $\mathrm{f} 0=$ & 91.90 \\
\hline & & $\mathrm{fb}=$ & 35.45 & $\mathrm{fb}=$ & 23.15 & $\mathrm{fb}=$ & 28.67 \\
\hline & & $\mathrm{k}=$ & 4.94 & $\mathrm{k}=$ & 7.42 & $\mathrm{k}=$ & 9.34 \\
\hline & & $\mathrm{R}^{2}=$ & 0.9999 & $\mathrm{R}^{2}=$ & 0.9994 & $\mathrm{R}^{2}=$ & 0.9999 \\
\hline & \multirow{4}{*}{ jardín ITS Villada } & $\mathrm{f} 0=$ & 36.64 & $\mathrm{f0}=$ & 94.61 & $\mathrm{fO}=$ & 70.41 \\
\hline & & $\mathrm{fb}=$ & 18.25 & $\mathrm{fb}=$ & 27.46 & $\mathrm{fb}=$ & 28.82 \\
\hline & & $\mathrm{k}=$ & 1.65 & $\mathrm{k}=$ & 10.22 & $\mathrm{k}=$ & 3.50 \\
\hline & & $\mathrm{R}^{2}=$ & 0.9967 & $\mathrm{R}^{2}=$ & 0.9989 & $\mathrm{R}^{2}=$ & 0.9994 \\
\hline & \multirow{4}{*}{ Torres de Junior } & $\mathrm{f} 0=$ & 74.20 & $\mathrm{f} 0=$ & 35.56 & $\mathrm{f} 0=$ & 87.35 \\
\hline & & $\mathrm{fb}=$ & 13.32 & $\mathrm{fb}=$ & 14.09 & $\mathrm{fb}=$ & 20.39 \\
\hline & & $\mathrm{k}=$ & 5.69 & $\mathrm{k}=$ & 7.32 & $\mathrm{k}=$ & 19.24 \\
\hline & & $\mathrm{R}^{2}=$ & 0.9963 & $\mathrm{R}^{2}=$ & 0.9950 & $\mathrm{R}^{2}=$ & 0.9992 \\
\hline
\end{tabular}


Tabla 2. Valores medios de los parámetros de Green \& Ampt y su rango de variación (entre paréntesis) según el uso del suelo

\begin{tabular}{c|cc|cc||cc}
\hline parámetros & \multicolumn{2}{|c|}{ Calles } & \multicolumn{2}{|c|}{ Parques } & \multicolumn{2}{c}{ Residencias } \\
\hline $\mathrm{f}_{0}(\mathrm{~mm} / \mathrm{h})$ & 67.8 & $(18.8-123.3)$ & 88.7 & $(36.4-208.6)$ & 68.8 & $(35.6-94.6)$ \\
$\mathrm{f}_{\mathrm{b}}(\mathrm{mm} / \mathrm{h})$ & 22.7 & $(12.5-36.6)$ & 30.4 & $(14.7-47.8)$ & 23.3 & $(13.3-35.5)$ \\
$\mathrm{k}(1 / \mathrm{h})$ & 9.9 & $(3.9-16.2)$ & 15.5 & $(4.0-51.1)$ & 7.7 & $(1.6-19.2)$ \\
\hline
\end{tabular}

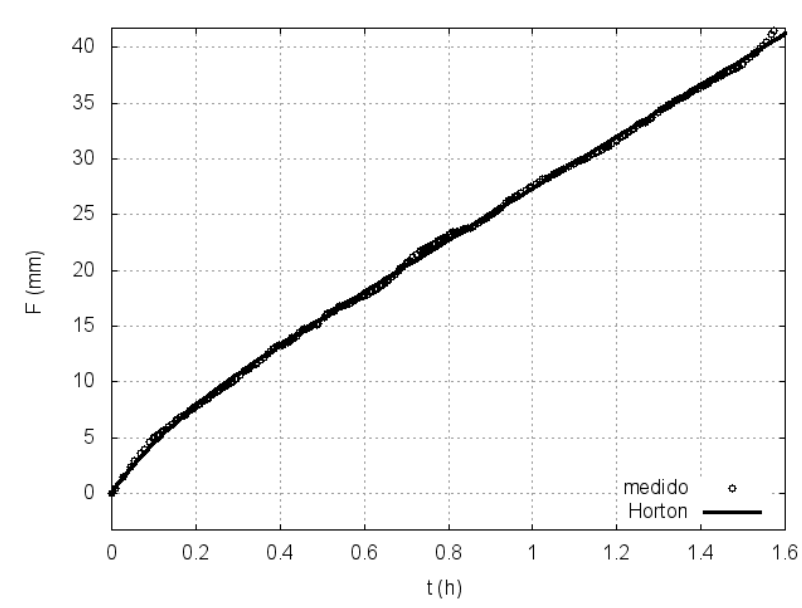

Figura 3. Infiltración acumulada medida y simulada por Horton - $2^{\circ}$ medición - casa $\mathrm{B}^{\mathrm{o}}$ Marqués de Sobremonte.

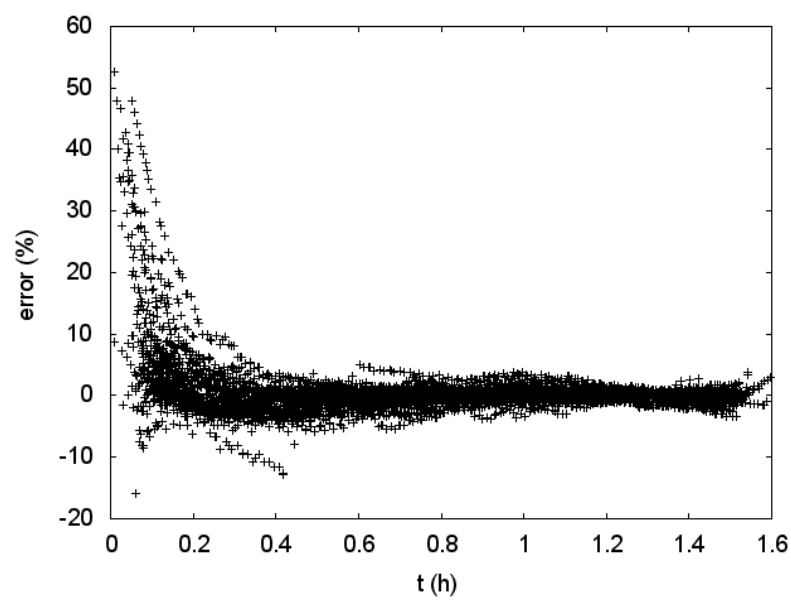

Figura 5. Error (en \%) en la estimación de la infiltración acumulada en función del tiempo de ensayo.

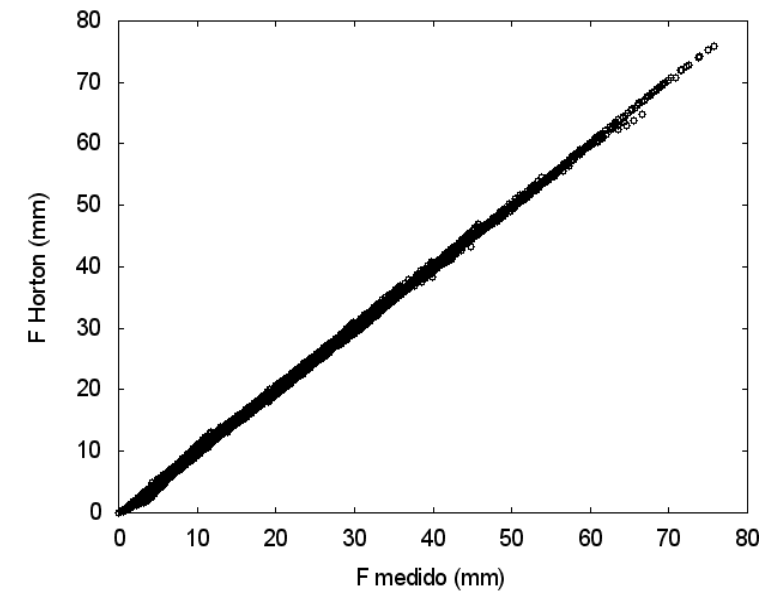

Figura 4. Infiltración acumulada medida versus simulada por Horton.

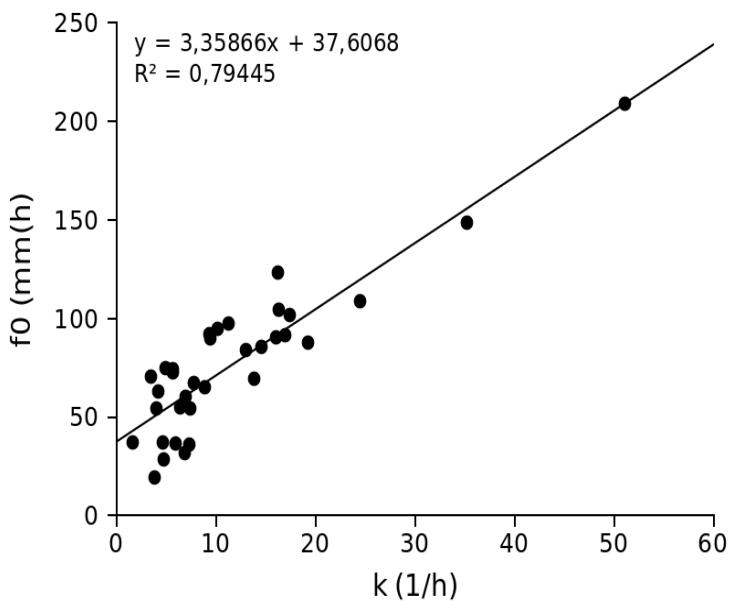

Figura 6. Relación entre parámetros $f_{0}$ y $k$. 


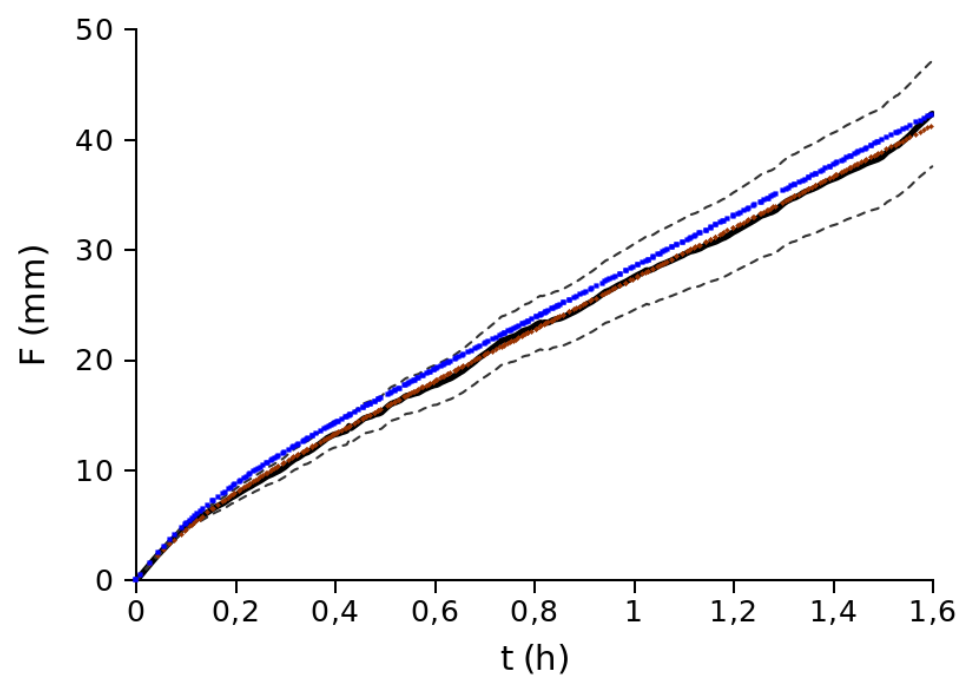

Figura 7. Infiltración medida (línea negra), su banda de incertidumbre (línea de trazo), valores predichos por Horton a 3 parámetros (puntos rojos) o con la Ecuación 6 (puntos azules).

\section{CONCLUSIONES}

A partir de la información recopilada in situ durante la campaña realizada en diversos sitios de la ciudad de Córdoba, ha sido posible ajustar los parámetros del modelo de infiltración de Horton. En promedio, los sitios correspondientes a parques y paseos públicos presentaron los mayores valores en los tres parámetros del modelo, frente a los jardines residenciales y las calles de tierra. Los mayores errores porcentuales en la estimación de la infiltración ocurren para valores bajos de la misma, por tanto al comienzo del proceso. Ha sido posible detectar una cierta dependencia entre los parámetros $f_{0}$ y $k$, cuyo ajuste por regresión lineal da lugar a una expresión que permitiría reducir a dos el número de parámetros libres del modelo, con cierto sacrificio en la calidad de los resultados. A pesar de ello, estos errores son compatibles con los de la propia lluvia generada, y por tanto, con los de la infiltración medida, lo que permitiría utilizar la Ecuación 6 como un estimador del valor de $f_{0}$ a partir de $k$ (o viceversa).

En cualquier caso, se considera que el conjunto de parámetros así ajustados resultará de interés para los proyectistas vinculados al manejo del escurrimiento pluvial urbano en la ciudad.

\section{REFERENCIAS}

ACHUTEGUI, A.; ABREU X.; PÁEZ M. L. Diseño y evaluación de un simulador de lluvias tipo gotero con efecto vibratorio. Venesuelos, v. 4, p. 24-28, 1996.

AOKI A. M.; SERENO R. Modificaciones de un microsimulador de lluvia. Investigación Agrícola, v. 19, n. 1/2, p. 47-51, 1999.

BENITO, E.; SANTIAGO, J. L.; DE BLAS, E.; VARELA, M. E. Deforestation of waterrepellent soils in Galicia (NW Spain): effects on surface runoff and erosion under simulated rainfall. Earth Surface Processes and Landforms, v. 28, p. 145-155, 2003. http://dx.doi.org/10.1002/esp.431 
CHOW, V. T.; MAIDMENT, D. R.; MAYS, L. W. Hidrología aplicada. Santafé de Bogotá: McGraw-Hill Interamericana, 1994.

EATON, J. W.; BATEMAN, D.; HAUBERG, S. GNU Octave: a high-level interactive language for numerical computations. Disponible en: www.octave.org. Acceso en : 2007.

HORTON, R. E. Analysis of runoff-plot experiments with varying infiltration capacity. Transactions American Goephysical Union, Washington, p. 693-711, 1939.

IRURTIA C. B.; MON R. Microsimulador de lluvia para determinar infiltración a campo. Publicación No 176. Buenos Aires: Instituto de Suelos, 1994. p. 18.

KAMPHORST, A. A Small rainfall simulator for the determination of soil erodibility. Netherlands Journal of Agricultural Science, v. 35, p. 407 - 415, 1987.

MATHEWS, J. H.; FINK, K. D. Numerical methods using Matlab, 4th Ed. [S.1.]: Pearson Prentice Hall, 2004.

QUINTANA SALVAT, F.; BARBEITO, O. Base geológica - geomorfológica para la planificación territorial de la ciudad de Córdoba y su entorno - Ejido Municipal. Fotointerpretación, v. 2, n. 1, p. 209-256, 1994.

RAVI, V.; WILLIAMS, J. R. Estimation of infiltration rate in the vadose zone: compilation of simple mathematical models. Volume I. Technical Report No. EPA/600/R-97/128a . Ada: United States Environmental Protection Agency; National Risk Management Research Laboratory, 1998.

TUCCI, C. E. M. Urban drainage in humid tropics. In: MAKSIMOVIC, C. Urban drainage in specific climates. Paris: UNESCO, 2001. Vol. 1. (IHP-V - Technical Documents in Hydrology, 40).

WEBER, J. F.; URBANO, J. M.; STUYCK, E. E.; AZELART, D.; MARTÍNEZ, N. B. Caracterización de los parámetros del modelo de Infiltración de Horton en suelos de la ciudad de Córdoba. Cuadernos del Curiham, v. 11, n. 1, p. 29-38, 2005.

WEBER, J. F.; PAOLI, H. I.; APESTEGUI, L. Microsimulador de lluvia portátil para estudios hidrológicos. Tecnología y Ciencia, año 9, n. 18, p. 47-53, 2010. 